\title{
Solenoid and sextupole optics of ultra-cold atomic hydrogen beams
}

\author{
W.A. Kaufman \\ Randall Laboratory of Physics, University of Michigan, Ann Arbor, Michigan 48109-1120, USA
}

Received 11 January 1993

Analytic calculations and particle tracking simulations are presented for a polarized atomic hydrogen beam produced by extraction from a superfluid helium coated cell in a large solenoidal magnetic field. Compression of the beam by the solenoidal field and subsequent focusing by a sextupole are considered.

\section{Introduction}

There is a strong motivation in high energy physics to develop high intensity polarized sources and targets. Since the recent demonstration of resonance suppression [1] in synchrotrons by the use of "snakes", the usual difficulties that hinder the acceleration of polarized beams may be largely mitigated. A high intensity polarized source could therefore extend collider experiments to include polarization physics without excluding users of unpolarized beams during periods of polarized beam acceleration.

Spin effect in proton-proton collisions are now usually investigated with solid cryogenic polarized proton targets and external polarized proton beams [2]. A polarized proton gas jet however could be used as an internal target in the circulating beam of a storage ring. Since it contains only hydrogen, such a target would eliminate the problem of discriminating against the background from the bound nucleons in a conventional solid target. This would simplify the study of exclusive reactions, and is essential to the study of inclusive reactions. Present techniques [3] for the production of free atomic beams give a maximum beam thickness of around $10^{12}$ atoms $/ \mathrm{cm}^{2}$. This is too small by two orders of magnitude to provide an adequate event rate in many high energy experiments.

Some relatively new techniques [4] for forming polarized atomic beams rely on the use of stabilized electron-spin polarized hydrogen [5]. Hydrogen atoms with their electron spins aligned interact via the ${ }^{3} \Sigma_{u}$ molecular pair potential. This potential supports no bound states, so that recombination to molecular hydrogen can be effectively suppressed. At high magnetic field $(\geq 5 \mathrm{~T})$ and low temperatures $(\leq 0.5 \mathrm{~K})$ the spectroscopic energy is much larger than the kinetic energy, which makes possible the separation of the four lowest lying hyperfine states into gases of elec- tron-spin polarized high field $\left(m_{\mathrm{s}}=-\frac{1}{2}\right)$ and low field $\left(m_{\mathrm{s}}=+\frac{1}{2}\right)$ seeking atoms. Lifetime enhancement of an atomic hydrogen gas also usually requires a confining surface that does not lead to rapid recombination. A superfluid film of helium is ideal for this purpose, since ${ }^{4} \mathrm{He}$ has a very small adsorption energy $\left(\epsilon_{\mathrm{a}} / k_{\mathrm{B}} \simeq 1 \mathrm{~K}\right)$ for hydrogen. The short dwell time of atomic hydrogen on the helium film makes the surface largely unavailable for use as the third body required for energy momentum conservation in the recombination process. Electron-spin polarized atomic hydrogen has been accumulated to densities greater than $10^{16}$ atoms $/ \mathrm{cm}^{3}$ without mechanical compression [6] in magnetic bottle storage cells. Assuming that the extraction efficiency can be made great enough, it is immediately clear that ultra-cold spin-stabilized hydrogen holds the promise of improving the density of present polarized atomic beams.

The purpose of this paper is to analyze the transport and magnetic focusing properties of a beam of spin polarized atomic hydrogen. The initial formation of such a beam will simply be assumed; questions of beam formation are beyond the scope of this work. A short review of the lowest hydrogen hyperfine states and their motion in a magnetic field is given in section 2. In section 3 the transport of a hydrogen beam formed in the gradient of a large solenoidal field and subsequently focused by a sextupole field is modeled analytically. The analytic model, useful in its own right, also serves as a check on a more realistic particle tracking simulation provided in section 4 . Section 5 is reserved for some final comments.

\section{Motion of the hydrogen ground state spin multiplet in a magnetic field}

Two interactions that lift the spin degeneracy of the ground state multiplet are the Zeeman and Fermi 
contact hyperfine interactions, given by

$H=H_{\mathrm{Z}}+H_{\mathrm{hyp}}=-\left(-g_{\mathrm{e}} \mu_{\mathrm{B}} s+g_{\mathrm{n}} \mu_{\mathrm{n}} i\right) \cdot \boldsymbol{B}+a i \cdot s$.

The magnetic moments of the electron and nucleon are $\mu_{\mathrm{e}}=-g_{\mathrm{e}} \mu_{\mathrm{B}} s$ and $\mu_{\mathrm{N}}=g_{\mathrm{n}} \mu_{\mathrm{n}} i$ respectively. $g_{\mathrm{e}}$ and $g_{\mathrm{n}}$ are the corresponding $g$ factors, whereas $\mu_{\mathrm{B}}$ and $\mu_{\mathrm{n}}$ are the Bohr and nuclear magnetons. Note that $\mu_{\mathrm{e}}$ is oriented antiparallel to $s$, so that in a magnetic field the lowest atomic energy state will have the electronic magnetic moment parallel to the field, but the electron spin antiparallel. The hyperfine constant is denoted by $a$. In zero magnetic field the total angular momentum, $f=i+s$, is a good quantum number, while in high field $\left(B \gg a / \mu_{\mathrm{e}} \approx 507 \mathrm{G}\right)$ the spin projections $m_{\mathrm{s}}$ and $m_{1}$, and thus also $m_{\mathrm{f}}$, are good quantum numbers. Diagonalization of the Hamiltonian (1) gives the energies

$E_{a, c}=-\frac{1}{4} a \mp \frac{1}{2} a \sqrt{1+\left(\mu^{+} B / a\right)^{2}}$,

$E_{b, d}=\frac{1}{4} a \mp \frac{1}{2} \mu^{-} B$,

where $\mu^{ \pm}=g_{\mathrm{e}} \mu_{\mathrm{B}} \pm g_{\mathrm{n}} \mu_{\mathrm{n}} \simeq 2 \mu_{\mathrm{B}}$, and the labeling of the states $a$ through $d$ is in order of ascending energy. The energy eigenstates in the $\left|m_{\mathrm{s}} m_{1}\right\rangle$ basis are

$|a\rangle=\cos \theta|\downarrow \uparrow\rangle-\sin \theta|\uparrow \downarrow\rangle$,

$|b\rangle=|\downarrow \downarrow\rangle$,

$|c\rangle=\cos \theta|\uparrow \downarrow\rangle+\sin \theta|\downarrow \uparrow\rangle$,

$|d\rangle=|\uparrow \uparrow\rangle$,

where

$\sin \theta=\left\{1+\left(\mu^{+} B / a+\sqrt{1+\left(\mu^{+} B / a\right)^{2}}\right)^{2}\right\}^{-1 / 2}$.

The force, due to the gradient of the field, on the hyperfine state $|\mathrm{h}\rangle$ is

$\boldsymbol{F}_{\mathrm{h}}=-\boldsymbol{\nabla} E_{\mathrm{h}}=\mu_{\mathrm{h}}^{\text {eff }} \nabla B$,

where $\mu_{\mathrm{h}}^{\text {eff }}=-\partial E_{\mathrm{h}} / \partial B$. Specifically,

$\mu_{b, d}^{\text {eff }}= \pm \frac{1}{2} \mu^{-} \simeq \pm \mu_{\mathrm{B}}$,

$\mu_{a, c}^{\mathrm{eff}}= \pm \frac{1}{2} \mu^{+} / \sqrt{1+\left(a / \mu^{+} B\right)^{2}}$

$$
\simeq \pm \mu_{\mathrm{B}} / \sqrt{1+\left(a / 2 \mu_{\mathrm{B}} B\right)^{2}} .
$$

Note that, modulo the distinction between $\mu^{+}$and $\mu^{-}$, the effective magnetic moments of the $a$ and $c$ states do not approach the constant effective moments of the $b$ and $d$ states until the condition $B \gg a / \mu^{+}(\simeq 506$ G) is satisfied.

The force described by eq. (4) produces a motion of the state $|h\rangle$ that can be calculated through the Lagrangian

$L_{\mathrm{h}}=\frac{1}{2} m\left(\dot{\rho}^{2}+\rho^{2} \dot{\varphi}^{2}-\dot{z}^{2}\right)-U_{\mathrm{h}}(\rho, z)$,

where $m$ is the mass of the hydrogen atom, the kinetic energy has been written in terms of the cylindrical coordinates $(\rho, \varphi, z)$, and the potential energy $U_{\mathrm{h}}(\rho, z)=E_{\mathrm{h}}(B)$ has been assumed independent of $\varphi$.
(This independence holds for any solenoid or cylindrical $n$-pole field.) The Lagrangian yields the equations of motion

$$
\begin{array}{ll}
p_{\rho}=m \dot{\rho}, & \dot{p}_{\rho}=m \rho \dot{\varphi}^{2}+\mu_{\mathrm{h}}^{\text {eff }} \partial B / \partial \rho, \\
p_{\varphi}=m \rho^{2} \dot{\varphi}, & \dot{p}_{\varphi}=0, \\
p_{z}=m \dot{z}, & \dot{p}_{z}=\mu_{\mathrm{h}}^{\text {eff }} \partial B / \partial z .
\end{array}
$$

The angular momentum $p_{\varphi}$ is a constant of the motion since the moduli of the magnetic fields considered do not depend on $\varphi$. Therefore, the angular velocity can be written as a function of the radius:

$\dot{\varphi}=k / \rho^{2}=\rho_{0}^{2} \dot{\varphi}_{0} / \rho^{2}$,

where $\dot{\varphi}_{0}$ and $\rho_{0}$ are the initial values of angular velocity and radius. Inserting (8) into (7) provides the following second order equations of motion for the coordinates $\rho$ and $z$ :

$\ddot{\rho}=k^{2} / \rho^{3}+\left(\mu_{\mathrm{h}}^{\text {eff }} / m\right) \frac{\partial B}{\partial \rho}$,

$\ddot{z}=\left(\mu_{\mathrm{h}}^{\text {eff }} / m\right) \frac{\partial B}{\partial z}$.

The only notable distinction between eqs. (4) and (9) is the inclusion of the centrifugal barrier term in eq. $(9 \mathrm{a})$. An examination of the equations of motion (9) and the effective magnetic moments (5) indicates that states $a$ and $b$ are attracted to, while states $c$ and $d$ are repelled from high magnetic field regions by the field gradients.

\section{The analytic model}

The system to be modeled consists of an ultra-cold $(\sim 300 \mathrm{mK})$ beam of $c$ and $d$ state atoms effusing from an aperture in the field gradient of a solenoid, followed by a sextupole focal element. For simplicity, the aperture is taken to be a point source located somewhere in the solenoid gradient. The treatment of the solenoid field is radically simplified by considering only regions close to the solenoid axis, and by setting the axial gradient to a constant. Requiring that the solenoid field be sourceless yields the components

$B_{\rho}=\frac{1}{2} B^{\prime} \rho$,

$B_{z}=B^{\prime}\left(z_{2}-z\right), \quad z_{1}<z<z_{2}$.

Here, the axial gradient is $B^{\prime} \equiv-\mathrm{d} B_{z} / \mathrm{d}_{z}=B_{\mathrm{c}} / \Delta z$, where $B_{c}$ is the central field, and $\Delta z \equiv z_{2}-z_{1}$. The distances $z_{1}, z_{0}, z_{2}, z_{3}, z_{4}$, and $z_{\mathrm{f}}$ are measured from the solenoid center and, in this approximation, denote respectively the point at which the field begins to fall, the location of the point source, the point at which the solenoid field has vanished, the entrance and exit of 


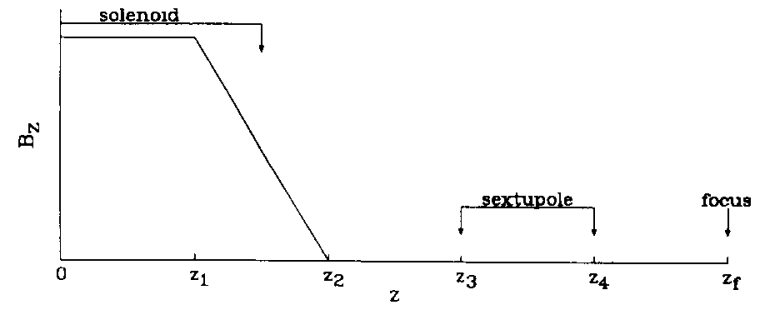

Fig. 1. The layout of the solenoid-sextupole system. The particular case of $z_{1}=7.5 \mathrm{~cm}, z_{2}=22.5 \mathrm{~cm}, z_{3}=45.5 \mathrm{~cm}$, $z_{4}=75.5 \mathrm{~cm}$, and $z_{\mathrm{f}}=95.5 \mathrm{~cm}$ is modelled analytically in the text.

the sextupole, and the final paraxial focus, as depicted in fig. 1. Using eq. (10), the non-zero components of $\nabla B$ are

$$
\begin{aligned}
& \frac{\partial B}{\partial \rho}=\frac{1}{4} B^{\prime 2} \rho \frac{1}{B}, \\
& \frac{\partial B}{\partial z}=-B^{\prime 2}\left(z_{2}-z\right) \frac{1}{B} .
\end{aligned}
$$

In order to arrive at simple approximate solutions to the equations of motion, it is assumed that $B \simeq B_{z}$ (i.e. $B_{\rho}$ is small near the $z$ axis), and that the $z$ dependence of the radial force (eq. (11a)) may be averaged over by replacing $z$ with $\left(z_{0}+z_{2}\right) / 2$. This procedure yields the expressions

$$
\begin{aligned}
& \frac{\partial B}{\partial \rho} \simeq \frac{1}{2} B^{\prime} \frac{\rho}{z_{2}-z_{0}}, \\
& \frac{\partial B}{\partial z} \simeq-B^{\prime},
\end{aligned}
$$

so that, for a point source in particular, the equations of motion become

$\ddot{\rho}=-\frac{\mu_{\mathrm{B}}}{2 m} B^{\prime} \frac{\rho}{z_{2}-z_{0}}$,

$\ddot{z} \simeq \frac{\mu_{\mathbf{B}}}{m} B^{\prime}$.

In eqs. (13) it was assumed that everywhere $B \gg 506$ $\mathrm{G}$, so that $\mu_{c, d}^{\text {eff }} \simeq-\mu_{\mathrm{B}}$. Eqs. (13a) and (13b) can be solved and the dependent variable eliminated, to give the radius as a function of $z$ :

$$
\begin{aligned}
\rho(z)= & 2\left(z_{2}-z_{0}\right) \frac{\dot{\rho}_{0}}{\dot{z}_{m}} \\
& \times \sin \left[\frac{1}{\dot{z}_{m}}\left(\sqrt{\dot{z}_{0}^{2}+\dot{z}_{m}^{2}\left(\frac{z-z_{0}}{z_{2}-z_{0}}\right)}-\dot{z}_{0}\right)\right],
\end{aligned}
$$

where the point source at $z_{0}$ is located in a field $B_{0}=B_{c}\left(z_{2}-z_{0}\right) / \Delta z$. Here, $\dot{z}_{m}$ is the axial velocity that might be gained in the solenoid field,

$\dot{z}_{m}=\sqrt{\frac{2 \mu_{\mathrm{B}} B_{0}}{m}}=\sqrt{\frac{2 \mu_{\mathrm{B}} B^{\prime}\left(z_{2}-z_{0}\right)}{m}}$, and the maximum possible final axial velocity is

$\dot{z}_{\mathrm{f}}=\sqrt{\dot{z}_{0}^{2}+\dot{z}_{m}^{2}}$.

Note that if the radial force is neglected entirely, the equations of motion produce parabolic trajectories. After the solenoid field, the $c$ and $d$ state trajectories are straight lines that traverse a drift space, $z_{2}<z<z_{3}$, until they enter the sextupole. A sharp edge approximation is assumed, so that no longitudinal components of the sextupole field are considered. The magnitude of this field depends on $\rho$ only,

$B=B_{6} \frac{\rho^{2}}{\rho_{6}^{2}}$,

so that only a radial force acts on the atoms. $B_{6}$ and $\rho_{6}$ denote the sextupole pole-tip field and radius, respectively. The equations of motion for the $d$ state atoms in a sextupole field are

$\ddot{\rho}=\frac{-2 \mu_{\mathrm{B}} B_{6}}{m \rho_{6}^{2}} \rho$,

$\ddot{z}=0$,

where $\mu_{d}^{\text {eff }}$ has again been approximated by $-\mu_{\mathrm{B}}$. This can easily be solved to yield the well known result

$\rho(z)=\rho_{\imath} \cos \kappa\left(\frac{z-z_{l}}{\dot{z}_{\imath}}\right)+\frac{\dot{\rho}_{l}}{\kappa} \sin \kappa\left(\frac{z-z_{l}}{\dot{z}_{l}}\right)$,

$\kappa=2 \mu_{\mathrm{B}} B_{6} / m \rho_{6}^{2}$.

The initial values $\rho_{l}, \dot{\rho}_{l}, z_{l}$, and $\dot{z}_{\imath}$ refer to the trajectory parameters at the sextupole entrance.

The motion of the $c$ state atoms in a sextupole field is not as simple; the dependence of $\mu_{c}^{\text {eff }}$ on the magnetic field can no longer be ignored since the condition $B \gg \mu^{+} / a$ does not hold for a large region within the sextupole. After the sextupole, the $c$ and $d$ trajectories are again straight lines.

Use of eqs. (14) and (19), the fact that the trajectories are straight lines in the drift spaces, and matching at the boundaries the various regions, allows a full description of the $d$ state trajectories emanating from a point source at $z_{0}$ and traversing the arrangement pictured in fig. 1 . It is also useful to consider an offset of the source by an amount $\rho_{0}$ from the $z$ axis. The resulting general trajectory is, for $\dot{\varphi}_{0}=0$,

$$
\begin{aligned}
\rho(z)= & \rho_{0} \cos \left[\frac{1}{\dot{z}_{m}}\left(\sqrt{\dot{z}_{0}^{2}+\dot{z}_{m}^{2}\left(\frac{z-z_{0}}{z_{2}-z_{0}}\right)}-\dot{z}_{0}\right)\right] \\
& +2\left(z_{2}-z_{0}\right) \frac{\dot{\rho}_{0}}{\dot{z}_{m}} \\
& \times \sin \left[\frac{1}{\dot{z}_{m}}\left(\sqrt{\dot{z}_{0}^{2}+\dot{z}_{m}^{2}\left(\frac{z-z_{0}}{z_{2}-z_{0}}\right)}-\dot{z}_{0}\right)\right], \\
z_{1} \leq & z_{0}<z \leq z_{2},
\end{aligned}
$$




$$
\begin{aligned}
& \rho(z)= \rho\left(z_{2}\right)+\left(z-z_{2}\right) \rho^{\prime}\left(z_{2}\right), \\
& z_{2}<z \leq z_{3}, \\
& \rho(z)= \rho\left(z_{3}\right) \cos \kappa\left(\frac{z-z_{3}}{\dot{z}_{f}}\right) \\
& \quad+\frac{\dot{z}_{f}}{\kappa} \rho^{\prime}\left(z_{2}\right) \sin \kappa\left(\frac{z-z_{3}}{\dot{z}_{f}}\right), \\
& z_{3}<z \leq z_{4}, \\
& \rho(z)=\rho\left(z_{4}\right)+\left(z-z_{4}\right) \rho^{\prime}\left(z_{4}\right), \\
& z_{4}<z,
\end{aligned}
$$

where $\rho^{\prime}\left(z_{l}\right)=\mathrm{d} \rho /\left.\mathrm{d} z\right|_{z=z_{1}}, i=2,3,4$.

Fig. 2 displays some $d$ state trajectories emanating from a point source for several different initial angles $\theta_{0}$. The parameters chosen for purposes of illustration were $B_{0}=6 \mathrm{~T}, B^{\prime}=1 \mathrm{~T} / \mathrm{cm}, B_{6}(\rho=8 \mathrm{~cm})=0.94 \mathrm{~T}$, $z_{0}=16.5 \mathrm{~cm}, z_{2}=22.5 \mathrm{~cm}, z_{3}=45.5 \mathrm{~cm}, z_{4}=75.5 \mathrm{~cm}$, and $z_{\mathrm{f}}=95.5 \mathrm{~cm}$. The initial velocity, $\bar{v}_{0}=9360 \mathrm{~cm} / \mathrm{s}$, was taken since it is the average velocity of atoms effusing from an aperture in a volume containing a $300 \mathrm{mK}$ atomic hydrogen gas. The sextupole field was fixed by requiring that it focus the paraxial trajectories $20 \mathrm{~cm}$ beyond the sextupole. Two characteristics of fig. 2 are noteworthy: the solenoid field acts to compress the trajectories by a large amount, while the sextupole focuses them, with a certain amount of resulting radial aberration at the paraxial focus.

If $c$ state trajectories are considered instead, the largest departures from those of the $d$ state occur in the sextupole field. The $c$ state equations of motion in a sextupole field are, for $\dot{\varphi}_{0}=0$,

$$
\ddot{\rho}=-\kappa^{4} \rho^{3} / \sqrt{(\kappa \rho)^{4}-(a / m)^{2}},
$$$$
\ddot{z}=0 \text {. }
$$

Eq. (21a) may be integrated once to yield $\dot{\rho}^{2}=\dot{\rho}_{l}^{2}+\sqrt{\left(\kappa \rho_{\imath}\right)^{4}+(a / m)^{2}}-\sqrt{(\kappa \rho)^{4}+(a / m)^{2}}$.

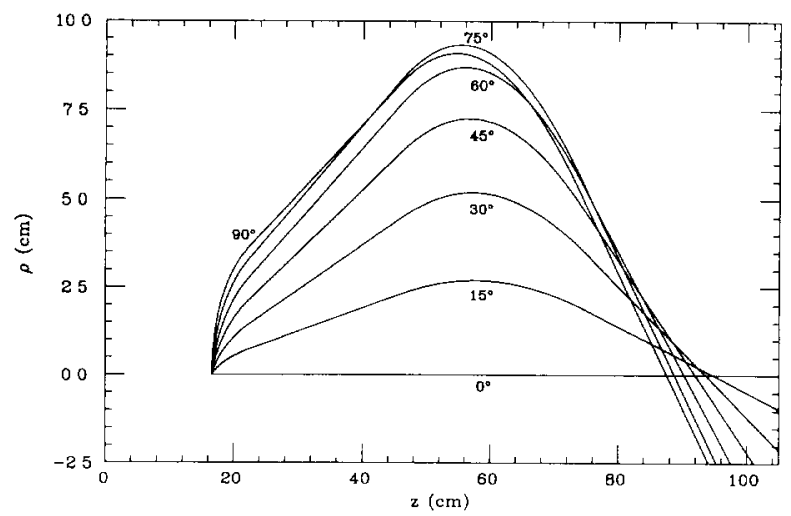

Fig. 2. The $d$ state trajectories' dependence on initial angle in $15^{\circ}$ increments.

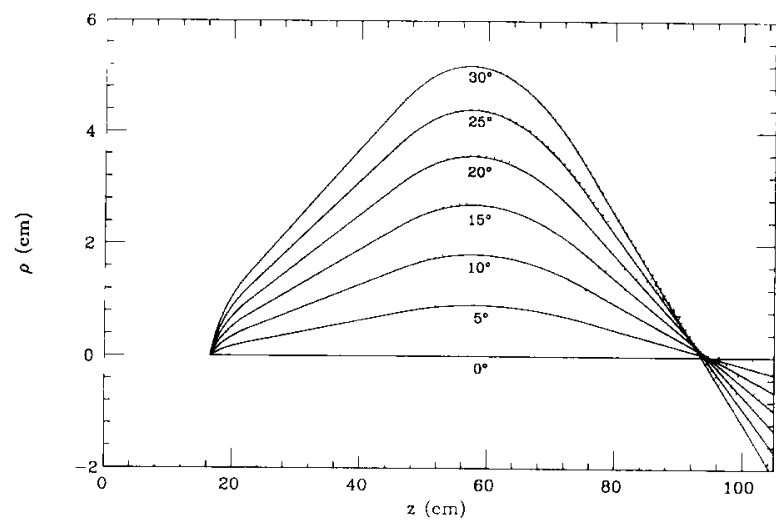

Fig. 3. The $c$ state (dotted curves) and the $d$ state (solid curves) trajectories' dependence on initial angle in $5^{\circ}$ increments.

Eq. (22) can be integrated once again to give the time or $z$ coordinate in terms of $\rho$-dependent elliptic integrals of the first and third kinds. However, for most purposes it is simpler to solve the first order equation (22) numerically; in this case the Burlisch-Stoer technique [7] was used. Fig. 3 displays the $c$ and $d$ state trajectories for the same parameters as in fig. 2, with initial angles between $0^{\circ}$ and $30^{\circ}$, in $5^{\circ}$ increments. It was assumed that the $c$ and $d$ trajectories from the source to the sextupole entrance are identical, since an appreciable difference in the trajectories only occurs where the field is small ( $B \leq 506 \mathrm{G}$ ) and the gradients large. At angles greater than $\sim 30^{\circ}$ the $c$ and $d$ trajectories nearly coincide in the sextupole, whereas for smaller initial angles the $c$ state is more weakly focused than the $d$ state. Some $c$ state trajectories $\left(\theta_{0} \leq\right.$ $5^{\circ}$ ) do not even return to cross the magnetic axis. Provided that only the smaller angles are accepted, the sextupole acts as a nuclear spin filter, with $d$ state atoms well focused in a relatively diffuse background of $c$ state atoms.

Fig. 4 shows the radial aberration $A^{\text {total }}\left(\theta_{0}\right)$ of the $d$ state trajectories at the paraxial focus $z_{\mathrm{f}}(0)$, as a function of initial angle $\theta_{0}$, for several different values of the initial field $B_{0}$. This aberration is given by the deviation of the crossing of the axis $z_{f}\left(\theta_{0}\right)$ from the paraxial crossing $z_{\mathrm{f}}(0)$, times the final slope of the trajectory:

$$
\begin{aligned}
& A^{\text {total }}\left(\theta_{0}\right)=\left(z_{\mathrm{f}}(0)-z_{\mathrm{f}}\left(\theta_{0}\right)\right)\left|\rho^{\prime}\left(z_{4}\right)\right|, \\
& z_{\mathrm{f}}\left(\theta_{0}\right)=z_{4}-\rho\left(z_{4}\right) / \rho^{\prime}\left(z_{4}\right) .
\end{aligned}
$$

The slope of the solenoid field was held fixed at $B^{\prime}=1$ $\mathrm{T} / \mathrm{cm}$, and $z_{2}$ was fixed at $22.5 \mathrm{~cm}$, so that the several values of $B_{0}$ in fig. 4 also correspond to different values of the initial position $z_{0}$ of the source. The sextupole field was adjusted so that the paraxial focus remained at $z_{\mathrm{f}}=95.5 \mathrm{~cm}$, regardless of the value of $B_{0}$. 


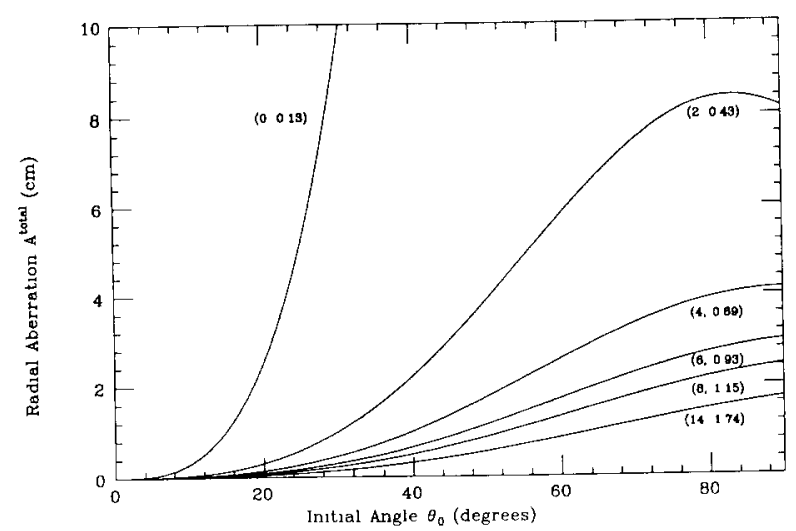

Fig. 4. The total radial aberration at the paraxial focus as a function of initial angle. The pairs of numbers indicate the solenoid field $B_{0}$ at the source and the sextupole field $B_{6}(\rho=$ $8 \mathrm{~cm}$ ) required to achieve the desired focus. The fields are in tesla.

The radial aberration introduced by the solenoid can also be written in a similar manner:

$$
\begin{aligned}
& A^{\mathrm{sol}}\left(\theta_{0}\right)=\left(z_{0}-z_{c}\left(\theta_{0}\right)\right) \rho^{\prime}\left(z_{2}\right), \\
& z_{c}\left(\theta_{0}\right)=z_{2}-\rho\left(z_{2}\right) / \rho^{\prime}\left(z_{2}\right) .
\end{aligned}
$$

This is displayed in fig. 5 which indicates that the solenoid aberration increases with increasing solenoid field $B_{0}$. However, fig. 4 shows that for the solenoidsextupole system the total aberration decreases as the solenoid field is increased. This can be easily understood by ascribing the most important source of aberration in the full system to large angle effects within the sextupole; the higher the solenoid field, the more the beam is compressed prior to its arrival at the sextupole, thereby suppressing the total aberration.

In designing a target, the relevant figure of merit is the thickness, given by the hydrogen beam intensity divided by the height presented to the accelerator

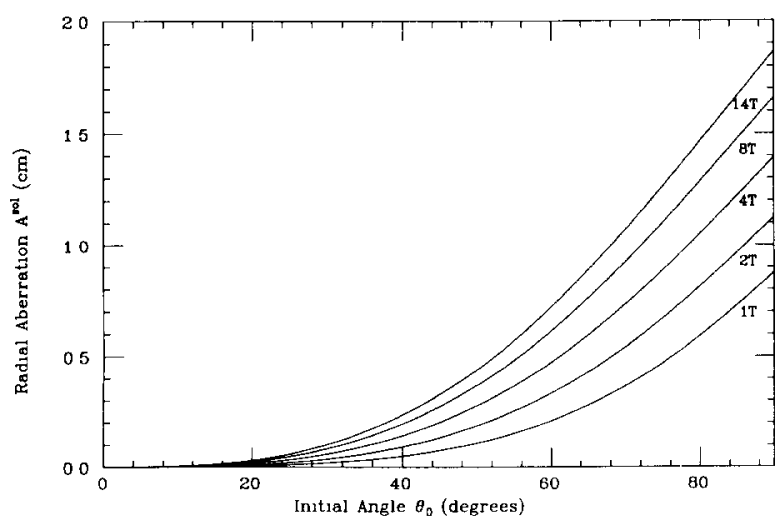

Fig. 5. The solenoid radial aberration as a function of initial angle. The field at the source is indicated. beam and the hydrogen beam velocity:

$T=I / h v$.

Eqs. (20) can be used to discover the optimal field in which to place the hydrogen beam source in order to maximize the target thickness. The result of the analytic model for $d$ state atoms with $B^{\prime}=1 \mathrm{~T} / \mathrm{cm}, z_{2}=$ $22.5 \mathrm{~cm}, z_{3}=45.5 \mathrm{~cm}, z_{4}=75.5 \mathrm{~cm}, z_{\mathrm{f}}\left(45^{\circ}\right)=95.5 \mathrm{~cm}$ is displayed in fig. 7. It was assumed that the hydrogen beam effuses from a $300 \mathrm{mK}$ point source, so that the distribution of initial angles was

$f\left(\theta_{0}\right)=2 \sin \theta_{0} \cos \theta_{0}$,

and the single initial speed used was the average speed

$\bar{v}_{0}=\frac{3}{4} \sqrt{2 \pi k T / m} \simeq 9360[\mathrm{~cm} / \mathrm{s}]$.

The height presented to the accelerator beam was taken as $0.5 \mathrm{~cm}$, to typify a high energy proton beam. Eqs. (20) and (26) were then used to calculate the percentage of atoms leaving the source that passed within $\rho=0.25 \mathrm{~cm}$ of the $z$ axis at $z_{\mathrm{f}}\left(\theta_{0}=45^{\circ}\right)=95.5$ $\mathrm{cm}$. ( $\theta_{0}=45^{\circ}$ was chosen, since this is the most probable initial angle for an atom in an effusing beam.) Dividing by the final velocity $\dot{z}_{\mathrm{f}}$ then yields the relative target thickness. Examination of the figure indicates that to maximize the target thickness, the source should be placed in a field of approximately $9 \mathrm{~T}$, for the various parameters chosen. The particular choice of parameters was influenced by the desire to keep the overall beam transport length short $(\leqq 100 \mathrm{~cm})$, so that intra-beam scattering and scattering from residual gas might be minimized. Also, the field of the $30 \mathrm{~cm}$ long sextupole required to focus the beam effusing from the source at $B_{0}=9 \mathrm{~T}$ is comparatively modest: $B_{6}(\rho=8$ $\mathrm{cm}) \simeq 1.2 \mathrm{~T}$. This makes magnetic shielding requirements relatively simple, and allows for use of either a conventional or superconducting magnet.

In the above analysis, neither finite source size effects (aperture aberrations) nor chromatic aberrations were considered. Because of the difficulty of producing an image to source magnification much less than one without increasing unrealistically both the length and diameter of the transport system, a source no larger than the desired image size $(\sim 0.5 \mathrm{~cm})$ will be investigated. The magnification may be estimated by calculating $\mathrm{d} \rho\left(z_{\mathrm{f}}\right) / \mathrm{d} \rho_{0}$ using eq. (20). For the set of parameters used earlier, a trajectory leaving a source placed at $B_{0}=9 \mathrm{~T}$, at an initial angle $\theta_{0}=45^{\circ}$, has a "magnification" $\mathrm{d} \rho\left(z_{\mathrm{f}}\right) / \mathrm{d} \rho_{0}=-0.91$, which indicates the source is imaged nearly one to one, and inverted. It therefore appears that the aperture size in this example is not a serious source of aberration. However, for a finite size source the centrifugal barrier in the radial equation of motion (eq. (9a)) must also be taken into account. (Both aperture and chromatic aberrations, as well as a realistic solenoid field, are fully included in 
the particle tracking simulation described in the next section.)

To investigate chromatic effects, the velocity spread of the beam must be included. The Maxwell distribution of speeds, modified for effusion, is

$F(v)=\frac{9}{2} \frac{v^{3}}{\tilde{v}^{4}} \mathrm{e}^{-3 t^{2} / 2 \tilde{i}^{2}}$,

$\tilde{v}=\sqrt{3 k T / m}$,

where $\tilde{v}$ is the most probable speed. The full width at half maximum for this distribution is approximately $\Delta v_{0}=0.95 \tilde{v}$. To estimate the extent by which the velocity spread shrinks due to acceleration in the solenoid field, one notes that $v_{\mathrm{f}}=\sqrt{v_{0}^{2}+\dot{z}_{m}^{2}}$, so that

$\Delta v_{\mathrm{f}}=\Delta v_{0} / \sqrt{1+\dot{z}_{m}^{2} / v_{0}^{2}}$.

For a $300 \mathrm{mK}$ source placed at $B_{0}=9 \mathrm{~T}$, one finds $v_{0} \equiv \bar{v}=85.7 \mathrm{~m} / \mathrm{s}, \Delta v_{0}=81.5 \mathrm{~m} / \mathrm{s}, v_{\mathrm{f}}=327.5 \mathrm{~m} / \mathrm{s}$, and $\Delta v_{\mathrm{f}}=21.3 \mathrm{~m} / \mathrm{s}$, so that the velocity spread has shrunk by almost a factor four, resulting in a very nearly monochromatic beam. The chromatic aberration at the focus can be estimated by noting that $\mathrm{d} \rho\left(z_{\mathrm{f}}\right) / \mathrm{d} v_{\mathrm{f}} \simeq \mathrm{d} \rho\left(z_{\mathrm{f}}\right) / \mathrm{d} \dot{z}_{\mathrm{f}}$, and again using eq. (20). A straightforward calculation yields, for a trajectory starting at $B_{0}=9 \mathrm{~T}, \rho_{0}=0$, with initial angle $\theta_{0}=45^{\circ}$ and the parameters used before, a chromatic aberration of $\Delta \rho\left(z_{\mathrm{f}}\right) / \Delta \dot{z}_{\mathrm{f}} \simeq 4.7 \times 10^{-4} \mathrm{~s}$. Setting $\Delta \dot{z}_{\mathrm{f}}$ equal to the half-width at half-maximum $\left(\Delta \dot{z}_{\mathrm{f}} \simeq 1065 \mathrm{~cm} / \mathrm{s}\right)$ gives a displacement $\Delta \rho\left(z_{\mathrm{f}}\right) \simeq 0.5 \mathrm{~cm}$. Therefore, one can conclude that the velocity spread of the beam remains a fairly serious source of aberration at the focus, even though the chromaticity was greatly improved by acceleration in the solenoid gradient. This suggests that perhaps a field somewhat greater than $9 \mathrm{~T}$ at the beam source aperture might be required to maximize the target thickness.

\section{The simulation}

Populations of atomic trajectories in the solenoidsextupole system were computed with a tracking simulation that numerically integrates the equations of motion using the Adams-Bashforth-Moulton "predictor-corrector" method [7]. The solution computed for the initial steps, via Runge-Kutta, is used to polynomially extrapolate (predict) the solution one step advanced; the extrapolation is then corrected using derivative information at the new point. An adaptive step size algorithm was used to keep the error within specified bounds. A substantial part of the tracking routine is based on a program written by Ellilä, Niinikoski, and Penttilä [8].

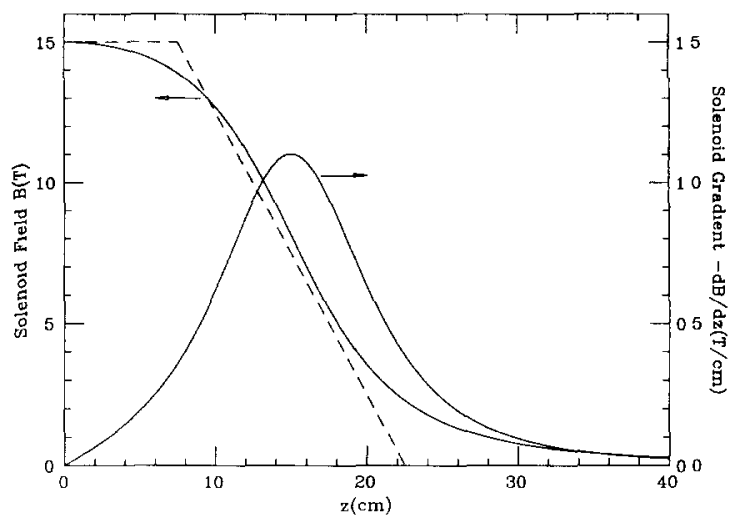

Fig. 6. The field and gradient along the axis of an infinitely thin solenoid of $30 \mathrm{~cm}$ length and $15 \mathrm{~cm}$ diameter. The dashed curve is the linear field used in the analytic modelling.

A realistic solenoid field and its derivatives can be produced numerically on a predetermined mesh by any number [9] of computer codes, and used as input to the tracking routine. However, to avoid any possible ambiguity in communicating the solenoid field adopted, we used instead the analytic expression [10] for the field of an infinitely thin solenoid of finite length. Local values of the field and its derivatives were provided by spline interpolation. To compare with the results of the analytic model, a solenoid field was chosen whose lowest order approximation is identical to the linear field employed earlier. The coil configuration consisted of an infinitely thin uniformly wound solenoid of $30 \mathrm{~cm}$ length and $7.5 \mathrm{~cm}$ radius. The axial field profile and its first derivative are displayed in fig. 6 .

The sextupole field was generated analytically within the tracking program, and a sharp edge approximation used. For simplicity, the sextupole field was assumed to be entirely shielded from dilution by the solenoid field. Its length and location were the same as those of the analytic model example of section 3 .

The initial data for the trajectories were generated by taking a random uniform deviate and, where feasible, applying the transformation method [7] to produce a random deviate with the desired distribution function. Otherwise, the rejection method [7] was employed. Specifically, the initial positions lay randomly and uniformly within a disc or rectangular area at a fixed axial position corresponding to the beam source aperture. The initial directions were chosen such that the azimuthal angles $\varphi_{0}$ were distributed uniformly between 0 and $2 \pi$, and the polar angles $\theta_{0}$ according to eq. (26). The initial speeds were distributed as described by eq. (28), with $T=300 \mathrm{mK}$. The resulting trajectories (typically 1000 per simulation) simulate an effusing beam of hydrogen atoms.

The isolate the effects of the various sources of aberration for a $d$ state beam, and to make an effective 


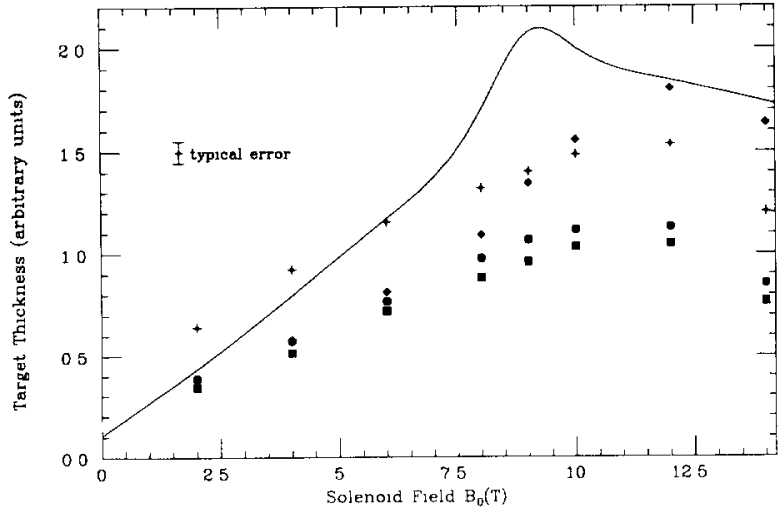

Fig. 7. The $d$ state target thickness as a function of the field at the source. The result of the analytic model, assuming a point source, effusive distribution of initial angle, and a single initial speed, is given by the curve. The points are the results of the tracking simulations using the solenoid field of fig. 6 . Diamonds - point source, effusive distribution of initial angle, a single initial speed. Circles - point source, effusive distribution of both initral angle and speed. Squares $-5 \mathrm{~mm}$ diameter source, effusive distribution of initial angles and speed. Crosses - $10 \times 2.5 \mathrm{~mm}^{2}$ rectangular source, effusive distribution of initial angles and speed.

comparison to the analytic model, the simulation was run succesively with

1) a monochromatic $\left(v_{0}=9360 \mathrm{~cm} / \mathrm{s}\right)$ point source with the angular distribution of eq. (26),

2) a point source with an effusive distribution of angles and speeds,

3) a $5 \mathrm{~mm}$ diameter source with effusion, and finally

4) a rectangular $10 \times 2.5 \mathrm{~mm}^{2}$ source with effusion.

The desired target region in all cases was a $5 \mathrm{~mm}$ diameter disc positioned $20 \mathrm{~cm}$ beyond the sextupole, except for the rectangular aperture, in which case the target was a $20 \times 5 \mathrm{~mm}^{2}$ rectangle. The transverse distributions were examined on target planes at various distances from the source aperture, and the sextupole field subsequently chosen to focus the beam in the target region.

Fig. 7 displays the results of the simulation for the four cases considered. The disparity between simulation 1) and the result of the analytic model is probably due to the crudeness of the linear approximation to the solenoid field. However, both show the expected maximum in target thickness because of the competition at higher solenoid field between increased transport efficiency from source to target and larger final beam velocity. Simulation 2) verifies the seriousness of the chromatic aberration that was found with the analytic model, while simulation 3 ) shows that the $5 \mathrm{~mm}$ diameter source size is not particularly troublesome for this example, as was also found in the analytic modelling. Simulation 4) demonstrates that some gain in thickness can be achieved by modifying the source and target sizes.

\section{Comments}

The target thickness units in fig. 7 are given by the fraction of the $d$ state beam transported to the target, divided by the final beam velocity in units of $\mathrm{cm} / \mathrm{s}$, multiplied by $10^{5}$. Therefore, for an initial $d$ state beam intensity of $10^{18}$ atoms $/ \mathrm{s}$, and a target height of $0.5 \mathrm{~cm}$, the simulations suggest that a target thickness of about $3 \times 10^{13}$ atoms $/ \mathrm{cm}^{2}$ could be obtained, if the placement of the source in the solenoid gradient is optimized. (Assuming effusive flow from a $5 \mathrm{~mm}$ diameter source at a temperature of $300 \mathrm{mK}$, a $d$ state intensity of about $10^{18}$ atoms $/ \mathrm{s}$ is expected if a density of $2 \times 10^{15}$ atoms $/ \mathrm{cm}^{3}$ is accumulated behind the source aperture.)

Simulations for $c$ state atoms indicated that the transport efficiency was typically about one-half that of the $d$ state.

No attempt was made in either the tracking simulations or the analytic modelling to include the acceptance of the sextupole or solenoid, i.e. no skimming of the beam by the finite diameter of the solenoid or sextupole was considered. It is clear that for a solenoid field at the source less than about $6 \mathrm{~T}$ (see fig. 2), and with reasonably dimensioned magnets, such considerations become increasingly important.

It is also possible to improve somewhat the transport efficiency from source to target by modifying the solenoid field with the introduction of a bucking coil at one end of the solenoid. This would increase the gradient and thereby the compression of the beam, and also remove the bulk of the unwanted field at large distances.

\section{Acknowledgements}

I would like to thank Professors G.R. Court, D. Kleppner, A.D. Krisch and Drs. R.S. Raymond, T. Roser and B. Vuaridel for useful discussions. This research was supported by the U.S. Dept. of Energy.

\section{References}

[1] A.D. Krisch et al., Phys. Rev. Lett. 63 (1989) 1137.

[2] R.C. Fernow and A.D. Krisch, Ann. Rev. Nucl. Part. Sci. 31 (1981) 109.

[3] P.A. Schmelzbach, D. Singy, W. Grüebler and W.Z. Zhang, Nucl. Instr. Meth. A251 (1986) 407.

[4] W.A. Kaufman, T. Roser and B. Vuaridel, University of Michigan preprint, May 1992; 
T. Roser, D.G. Crabb, W.A. Kaufman, R.S. Raymond, J.A. Stewart, B. Vuaridel and G.R. Court, Nucl. Instr. and Meth. A301 (1991) 42.

[5] I.F. Silvera and J.T.M. Walraven, in: Progress in Low Temperature Physics, Vol. X, ed. D.F. Brewer (NorthHolland, Amsterdam, 1986) pp. 139-370 (Chap. 3).

[6] J.T.M. Walraven, I.F. Silvera and A.P.M. Matthey, Phys. Rev. Lett. 45 (1980) 449;

R.W. Cline, D.A. Smith, T.J. Greytak and D. KJeppner, Phys. Rev. Lett. 45 (1980) 2117.

[7] W.H. Press, B.P. Flannery, S.A. Teukolsky and W.T.
Vetterling, Numerical Recipes: the Art of Scientific Computing (Cambridge University, 1989).

[8] M. Ellilä, T.O. Niinikoski and S. Penttilä, Nucl. Instr. and Meth. B14 (1986) 571.

[9] See for example M.T. Menzel and H.K. Stokes, Users Guide to the Poisson/Superfish Group of Codes, Los Alamos National Lab. publication LA-UR-87-115 (1987).

[10] E.E. Callaghan and S.H. Maslen, National Aeronautics and Space Administration, Cleveland, Ohio, Technical Note D-465 (1960). 